University of Nebraska - Lincoln

DigitalCommons@University of Nebraska - Lincoln

\title{
Ultrafast resonance-enhanced multiphoton ionization in the azabenzenes: Pyridine, pyridazine, pyrimidine, and pyrazine
}

Timothy D. Scarborough

University of Nebraska-Lincoln, tim.d.scarborough@gmail.com

David B. Foote,

University of Nebraska-Lincoln

Cornelis J. Uiterwaal

University of Nebraska - Lincoln, cuiterwaal2@unl.edu

Follow this and additional works at: https://digitalcommons.unl.edu/physicsuiterwaal

Part of the Physics Commons

Scarborough, Timothy D.; Foote,, David B.; and Uiterwaal, Cornelis J., "Ultrafast resonance-enhanced multiphoton ionization in the azabenzenes: Pyridine, pyridazine, pyrimidine, and pyrazine" (2012). C.J.G.J. Uiterwaal Publications. 17.

https://digitalcommons.unl.edu/physicsuiterwaal/17

This Article is brought to you for free and open access by the Research Papers in Physics and Astronomy at DigitalCommons@University of Nebraska - Lincoln. It has been accepted for inclusion in C.J.G.J. Uiterwaal Publications by an authorized administrator of DigitalCommons@University of Nebraska - Lincoln. 


\title{
Ultrafast resonance-enhanced multiphoton ionization in the azabenzenes: Pyridine, pyridazine, pyrimidine, and pyrazine
}

\author{
Timothy D. Scarborough, ${ }^{\text {a) }}$ David B. Foote, and Cornelis J. G. J. Uiterwaal \\ Department of Physics and Astronomy, University of Nebraska-Lincoln, Lincoln, Nebraska 68588-0299, USA
}

(Received 2 December 2011; accepted 18 January 2012; published online 7 February 2012)

\begin{abstract}
We report on the ultrafast photoionization of pyridine, pyridazine, pyrimidine, and pyrazine. These four molecules represent a systematic series of perturbations into the structure of a benzene ring which explores the substitution of a $\mathrm{C}-\mathrm{H}$ entity with a nitrogen atom, creating a heterocyclic structure. Data are recorded under intense-field, single-molecule conditions. The pulses (50 fs, $800 \mathrm{~nm}$ ) are focused into the molecular vapor, and ion mass spectra are recorded for intensities of $\sim 10^{13}$ $\mathrm{W} / \mathrm{cm}^{2}$ to $\sim 10^{15} \mathrm{~W} / \mathrm{cm}^{2}$. We measure ion yields in the absence of the focal volume effect without the need for deconvolution of the data. For all targets, stable singly- and doubly-charged parent ions $\left(\mathrm{C}_{6-\mathrm{n}} \mathrm{H}_{6-\mathrm{n}} \mathrm{N}_{\mathrm{n}}{ }^{+(+)}\right)$are observed with features suggesting resonance-enhanced ionization. From the intensity dependence of the ion yield, we infer that excitation occurs both through ${ }^{1} \pi \pi *$ transitions (remnants of the benzene structure) and through ${ }^{1} \mathrm{n} \pi^{*}$ transitions, the latter being a result of Rydberg-like excitations of the lone pair electrons of the nitrogen atoms. Stability against intensefield fragmentation is also discussed. @ 2012 American Institute of Physics. [doi:10.1063/1.3682541]
\end{abstract}

\section{INTRODUCTION}

Due to the considerable abundance and biological relevance of organic molecules similar to it, benzene has long been viewed as an archetypal molecule of organic chemistry. The stability of the aromatic six-member ring structure results in a wide variety of substituted variants in which the substitution of an atom or functional group can perturb the electronic structure while maintaining many of the features of benzene. ${ }^{1}$ For instance, functional groups can substitute for one or more hydrogen atoms and/or one or more nitrogen atoms may substitute for a carbon atom in the ring, making the molecule heterocyclic. Perhaps the most fundamental of these aromatic heterocycles, pyridine (azabenzene), involves the replacement of a single $\mathrm{C}-\mathrm{H}$ unit with a nitrogen atom; this alters the system but it remains isoelectronic with benzene. If this substitution is made twice, three separate isomers result depending on the relative positions of the two nitrogen atoms in the ring, as shown in Fig. 1: pyridazine (1,2-diazine), pyrimidine (1,3-diazine), and pyrazine (1,4-diazine).

Due to their fundamental similarity to benzene, their prevalence in herbicides ${ }^{2}$ and pharmaceutical drugs, ${ }^{3}$ and pyrimidine's function as the base structure for the DNA/RNA nucleotides, the electronic structure of the azabenzenes has been studied thoroughly. Transitions, energies, and symmetry assignments have been investigated through electron-energy loss experiments and UV absorption, ${ }^{4-7}$ zero-kinetic-energy (ZEKE) electron spectroscopy, ${ }^{8-11}$ and more recently, absorption of vacuum ultraviolet (VUV) synchrotron radiation ${ }^{12}$ (Refs. 4-7, and 12 include comprehensive tables of the available electronic transitions in the azabenzenes). A thorough summary of the work done through 1988 was presented by

\footnotetext{
a) Author to whom correspondence should be addressed. Electronic mail: tim.scarborough@huskers.unl.edu.
}

Innes et al. ${ }^{13}$ However, while the electronic structure has been extensively explored, little research has been done into the intense-field regime where the dynamics become considerably more complex due to its non-perturbative character. The ongoing interest in intense-field molecular dynamics (and the dynamics of ionization processes, in particular) (Refs. 14-16) is driven by their relevance in attosecond pulse generation, ${ }^{17}$ generation of short-wavelength radiation, ${ }^{18}$ and ultimately the desire to control chemical reactions. ${ }^{19}$ Unfortunately, although computational work can simulate quite accurately the electronic structures of these molecules, fully $a b$ initio simulations of intense-field molecular photodynamics are far beyond reach even for the most advanced supercomputers. ${ }^{20}$ This makes experimental investigations all the more crucial.

The present work focuses on how the nitrogen substitutions affect the intense-field dynamics of pyridine and the diazines. Because the lone pairs of the nitrogen atoms result in energy levels near to the two highest occupied $\pi$ orbitals (remnants of the benzene system), there is a highest occupied molecular orbital (HOMO) band containing the two benzenelike $\pi$ orbitals as well as one (pyridine) or two (the diazines) interacting lone-pair orbitals. While the benzene $\pi$ system is resonant with three 800-nm photons in the monosubstituted halobenzenes, ${ }^{21}$ the energies of these transitions are now not only perturbed by the substitutions, but they are also in direct competition with $\mathrm{n} \pi^{*}, \mathrm{n}$ (Rydberg), and $\mathrm{n} \sigma^{*}$ transitions. Further, even when a low-field transition is out of resonance with an integer number of photons, the intense-field Stark shift may force such a transition into a "dynamic resonance." 22

Presented here are the yields of singly- and doublyionized parent molecules as a function of laser intensity for pyridine, pyridazine, pyrimidine, and pyrazine. In each case, we observe signatures of resonance-enhanced multiphoton ionization (REMPI). The measurements as presented were taken in the absence of the focal volume effect; ${ }^{21,23}$ in 

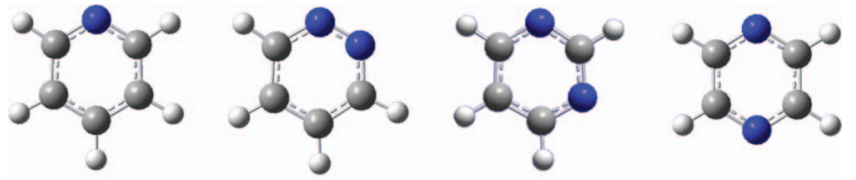

FIG. 1. The mono- and di-substituted azabenzenes. Pictured left to right: pyridine (azabenzene), pyridazine (1,2-diazine), pyrimidine (1,3-diazine), and pyrazine (1,4-diazine).

the presence of the volume effect, the features indicative of REMPI would not have been seen. Unlike other methods to avoid the volume effect, ${ }^{24-27}$ our method allows direct measurement with three-dimensional spatial resolution without the need for further deconvolution or assumptions about beam quality.

Our measured ion yields are presented in Fig. 2, in which the ion yield $Y$ is plotted against the pulse intensity $I$ with both axes on logarithmic scales. This double-logarithmic representation is customary and convenient in studies of intense-field ionization, because ion yields from multiphoton processes rise as $Y \propto I^{m}$, where $m$ is the number of photons involved. ${ }^{28}$ In the double-logarithmic representation, these yields appear as straight lines with slope $m$. Based on our estimates of the Keldysh parameter, ${ }^{29}$ multiphoton ionization is likely; this is ultimately supported by the existence of several such straight lines with integer slope in our experimental data. Fitted values for the slopes are presented in Table I. For each target molecule we find two regions of intensities with integer slope, a signature of REMPI. In this process a resonant transition to an excited molecular orbital becomes saturated such that a

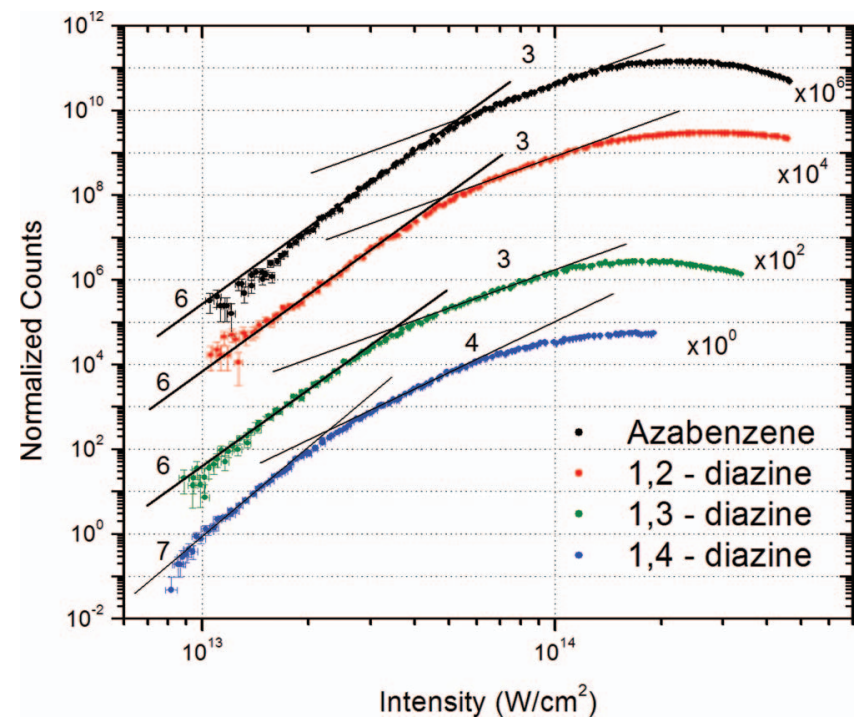

FIG. 2. Molecular parent ion yields of pyridine (azabenzene) in black, pyridazine (1,2-diazine) in red, pyrimidine (1,3-diazine) in green, and pyrazine (1,4-diazine) in blue as a function of intensity, measured with $800 \mathrm{~nm}, 50 \mathrm{fs}$ pulses. Data have been normalized for consistency in pressure and collection time, and have been shifted vertically by two orders of magnitude per dataset to allow viewing on the same graph. Errors in the count rate are based on counting statistics, while errors in intensity are estimated from experimental uncertainties in power measurement and laser stability. Thin black lines marked with the log-log slope (and thus the number of photons in the process) are meant to guide the eye, and represent our determination of the most accurate integer slope. Mathematically fitted slope values can be found in Table I.
TABLE I. Mathematically fitted slopes. Fitted slopes were determined by plotting $\log$ (yield) vs. $\log$ (intensity) and fitting to linear trends weighted by the error bars over the relevant regions. The range over which a slope was fitted was determined by finding the limits where, by including more data points of higher or lower intensity, the slope no longer remained consistent, whereas removing points did not significantly change the slope.

\begin{tabular}{lcc}
\hline \hline Species & Low-intensity slope fit & High-intensity slope fit \\
\hline Pyridine & $5.99 \pm 0.05$ & $3.04 \pm 0.01$ \\
Pyridazine & $5.99 \pm 0.07$ & $2.92 \pm 0.01$ \\
Pyrimidine & $6.01 \pm 0.08$ & $2.90 \pm 0.01$ \\
Pyrazine & $6.84 \pm 0.15$ & $4.05 \pm 0.02$ \\
\hline \hline
\end{tabular}

smaller number of photons can ionize the target from this almost immediately populated excited state. At the highest intensities used, the parent ion yields of all species decrease as other processes become dominant, such as multiple ionization and fragmentation.

\section{RESULTS AND DISCUSSION}

\section{A. Parent ion yields}

Parent ion yields of all four species are seen in Fig. 2. In this representation, each curve is separated from the next by factors of 100 for clarity; on a log-log plot this merely shifts the curve without altering it. The curve for pyridine, when fit to two regions with integer log-log slope, displays a slope of six at low intensity giving way to a slope of three at higher intensity, indicative of a $(3+3)$ REMPI process. Six-photon ionization is expected when considering ionization from either the HOMO or HOMO-1, which are nearly isoenergetic $\pi$ and $n$ orbitals (the $n$ orbital strongly correlating to the lone pair of the substituent nitrogen atom), as only these orbitals are within the range of six 800-nm photons (although only barely). ${ }^{4}$ Of these two orbitals, only the $\pi$ has a single-photon transition energetically resonant with three $800 \mathrm{~nm}$ photons necessary to observe $(3+3)$ REMPI. In this case, the excitation mechanism is the ${ }^{1} \pi \pi *$ transition analogous to the $e_{1 g}^{4} e_{2 u}^{0} \rightarrow e_{1 g}^{3} e_{2 u}^{1}$ transition in benzene. These benzene-like structures prove to be fairly robust throughout this molecular series; the most relevant in this case is the ${ }^{1} \pi \pi * \mathrm{~S}_{0} \rightarrow \mathrm{S}_{1}$ in benzene (vertical energy $4.9 \mathrm{eV}$ ). In the molecules considered here, this transition is only slightly perturbed to vertical values between 4.8 and $5.1 \mathrm{eV}^{4-7}$

Upon close examination of pyridine in Fig. 3, however, it is apparent that at the lowest intensities the data trend slightly, but definitively, beneath a line with slope six. In fact, when fitting only the 29 lowest intensity data points, a slope of seven is found, followed by a slope six from points 29-55, and again the slope three from points 73-94 before higher order processes (multiple ionization, fragmentation) bring the parent yield down. Alternatively, if all of the first 55 points are fitted together, one finds a slope of 6.27; however, when this fit is applied, it becomes clear that this slope is too low for the slope-seven region and too high for the slope-six region. This makes it a poor overall compromise, since no range of intensities exists in which a slope 6.27 is a good fit. Due to the experimental uncertainties at low intensity (particularly low 


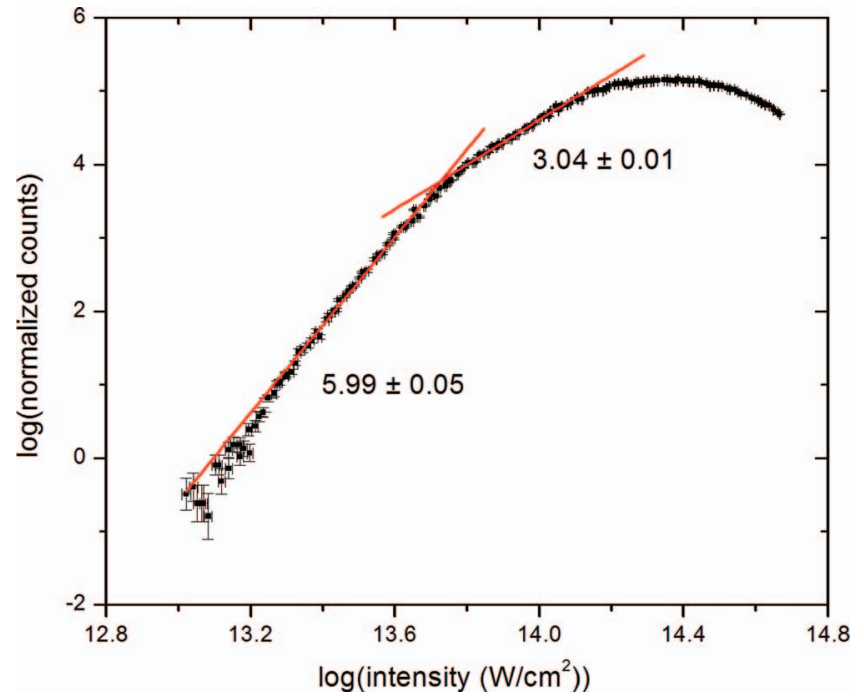

FIG. 3. Mathematically fitted slopes of pyridine. Plotting the $\log$ (yield) against the $\log$ (intensity) results in regions of integer linear slopes corresponding to $(3+3)$ resonance-enhanced multiphoton ionization (REMPI). Here the resonance enhancement comes from a ${ }^{1} \pi \pi^{*}$ transition analogous the $\mathrm{S}_{0} \rightarrow \mathrm{S}_{1}$ transition in benzene.

count rate and precise calibration of the intensities) we conclude that the slope of six is legitimate. ${ }^{30}$

Pyridazine, seen in Fig. 4, also exhibits log-log slopes of six and three, again indicative of $(3+3)$ REMPI. Although this result is the same as the curve for pyridine in a qualitative sense, the dynamics appears to be different. The introduction of the second nitrogen substituent creates an energetic splitting of their two lone pair states which owes to the symmetric $\left(\mathrm{n}_{+}\right)$and antisymmetric $\left(\mathrm{n}_{-}\right)$superpositions. Because the lone pairs in this case are adjacent, there is substantial spatial overlap which leads to a larger splitting than in the other diazines.

The energy difference between the HOMO and all other bound states leaves the $\mathrm{n}_{-}$as the only orbital capable of supporting six-photon ionization, so the ionization dynam-

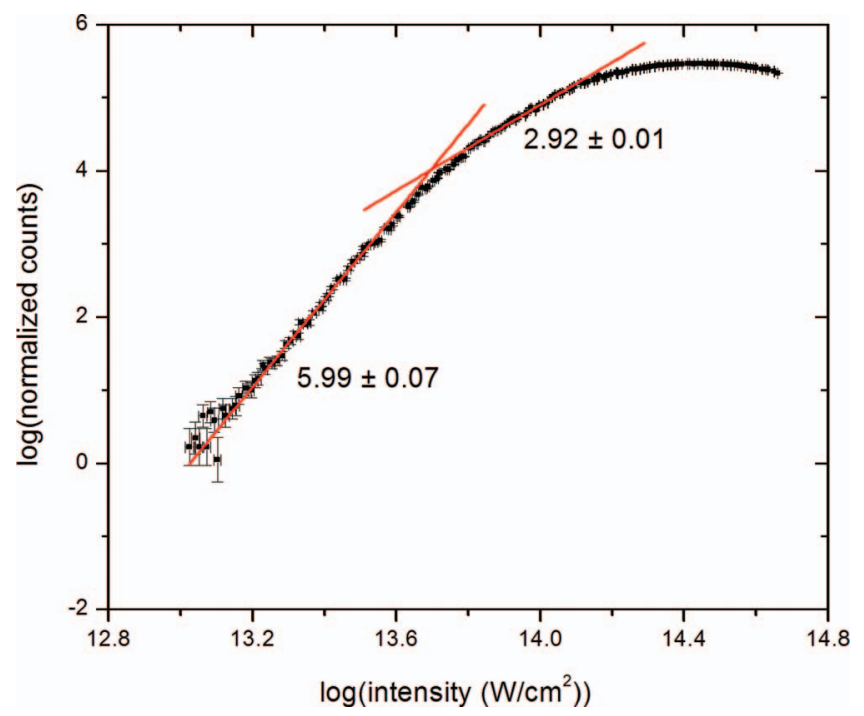

FIG. 4. Mathematically fitted slopes of pyridazine. Plotting the $\log$ (yield) against the $\log ($ intensity) results in regions of integer linear slopes corresponding to $(3+3)$ REMPI. Unlike pyridine, the dynamics in pyridazine appears to involve a ${ }^{1} \mathrm{n} \pi *$ excitation.

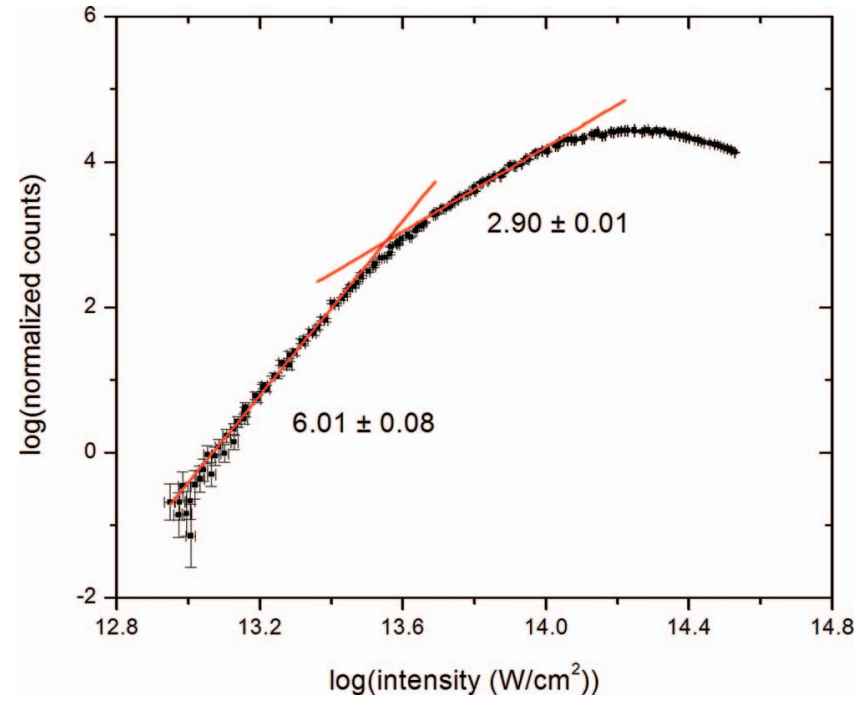

FIG. 5. Mathematically fitted slopes of pyrimidine. Plotting the $\log$ (yield) against the $\log$ (intensity) results in regions of integer linear slopes corresponding to $(3+3)$ REMPI. Like pyridazine, it appears that ionization in pyrimidine occurs following a ${ }^{1} \mathrm{n} \pi *$ excitation.

ics must originate there. But although the electronic excitations in pyridazine have been well documented ${ }^{5,31}$ there are no transitions to $\mathrm{n} \pi *$ or $\mathrm{n}$ (Rydberg) excited states that are on (or even near) resonance for excitation by three $800-\mathrm{nm}$ photons. At present this issue remains unresolved unless the vertical and adiabatic binding energies of the HOMO-1 (of $\pi$ structure) differ by more than $\approx 1.1 \mathrm{eV}$ (in which case the benzene-like ${ }^{1} \pi \pi^{*}$ structure could again be responsible for $(3+3)$ REMPI). However, such a large difference between the vertical and adiabatic energies is unlikely. ${ }^{32}$ At present we hypothesize that the ac Stark shift of the $n \pi^{*}$ state is significant enough to bring the transition into a dynamic resonance, ${ }^{33}$ since the field is large enough at these intensities to induce ponderomotive shifts ${ }^{28}$ of several $\mathrm{eV}$. Unfortunately, we must speculate here; the definitive dynamics in pyridazine remains open to interpretation.

Pyrimidine is also most accurately fit with log-log slopes of six and three, as seen in Fig. 5. In this case the dynamics must originate from the HOMO, here the antisymmetric $\mathrm{n}_{-}$, since it is the only orbital within the energetic range of six photons (although only barely; the first IP of pyrimidine is $9.342 \mathrm{eV}$ according to ZEKE measurements, ${ }^{10}$ where six 800 -nm photons are 9.30 with a spread of $0.06 \mathrm{eV}$ due to the pulse bandwidth). Here a ${ }^{1} \mathrm{n} \pi *$ transition (labeled ${ }^{1} \mathrm{~A}_{2}$ in Ref. 12) is most likely responsible for the dynamics. This transition has been observed in VUV absorption ${ }^{34}$ and electron-energy loss spectra ${ }^{6}$ at a vertical energy of 4.6-4.7 $\mathrm{eV}$. The multiphoton processes we typically observe seem to be restricted by adiabatic energies (three photons absorption up to $4.65 \mathrm{eV}$ dominates the excitation in benzene ${ }^{21}$ in spite of a well-documented vertical energy of $4.9 \mathrm{eV}^{5}$ ). Nevertheless, we find it reasonable to envision either a Stark shift of the levels involved in this transition or excitation to a vibrationally excited state such that a three-photon ${ }^{1} \mathrm{n} \pi$ * excitation could find itself on resonance.

The curve for pyrazine, seen in Fig. 6, shows slopes of seven and four, indicative of either $(3+4)$ or $(4+3)$ 


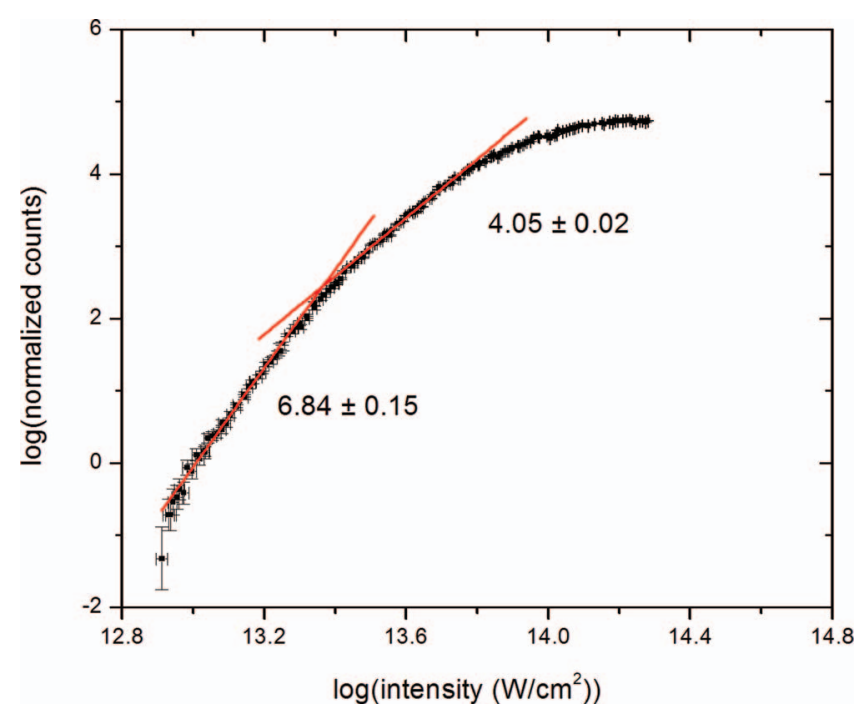

FIG. 6. Mathematically fitted slopes of pyrazine. Plotting the $\log$ (yield) against the $\log$ (intensity) results in regions of integer linear slopes corresponding to $(3+4)$ REMPI. Like pyridine, resonant excitation appears to occur through a ${ }^{1} \pi \pi^{*}$ transition analogous the $S_{0} \rightarrow S_{1}$ transition in benzene. Here the ground state is more deeply bound, so a seventh photon is required to ionize.

REMPI. The low-intensity slope is seven, although the HOMO $\left(\mathrm{n}_{+}\right)$should be within the reach of six photons (ZEKE measurements ${ }^{11}$ find it to be $9.309 \mathrm{eV}$; six $800-\mathrm{nm}$ photons is $9.30 \mathrm{eV}$ ). This would make the likeliest ionization a $(3+4)$ REMPI in the benzene-like $\pi$ system once again. In fact, even if the $n_{+}$orbital is also in the seven-photon range, electron-energy loss and single-photon experiments indicate that the benzene-like $\pi \pi^{*}$ transitions tend to dominate over the much weaker $\mathrm{n} \pi *$ transitions. ${ }^{7}$

We note that a part of the benzene structure includes transitions expected to be nearly resonant with four photons which could contribute to a $(4+3)$ REMPI process. We consider in principle all energetically allowed transitions, such as the $\pi \pi^{*}\left({ }^{1} \mathrm{~B}_{1 \mathrm{u}}\right)$ and $\mathrm{n} 3 \mathrm{~s}\left({ }^{1} \mathrm{~A}_{\mathrm{g}}\right)$, even those which are typically forbidden by symmetry in the single-photon case; calculating the transition matrix elements for multiphoton processes ${ }^{35}$ is prohibitively involved, and the complexity of multiphoton interaction can lead to "forbidden" single-photon transitions being allowed in the multiphoton case. However, a population rate model based on generalized cross sections with $I^{m}$ optical pumping rates in multiphoton absorption ${ }^{28}$ demonstrates that, if both three- and four-photon excitations are resonant, the three-photon channel dominates the excited state population. Even for similar generalized cross sections ${ }^{28}$ the threephoton resonant state acquires the majority of the probability density. In other words, a three-photon transition saturates at lower intensity that a four-photon transition. As a result, we determine that the $(3+4)$ REMPI is the leading source of parent ionization.

\section{B. Parent stability and muliply-charged parent ion yields}

As seen in Fig. 7, the parent ion yields differ significantly in their total yields near saturation. At each

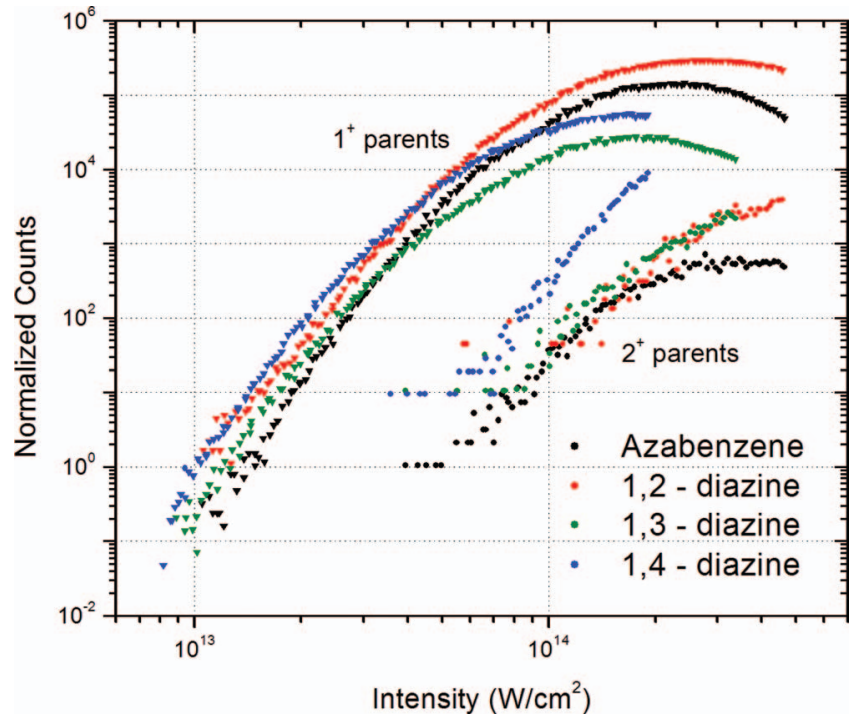

FIG. 7. Singly and doubly charged parent ions of pyridine (azabenzene) in black, pyridazine (1,2-diazine) in red, pyrimidine (1,3-diazine) in green, and pyrazine (1,4-diazine) in blue. All curves have been normalized for integration time and pressure fluctuations.

molecule's saturation intensity, it is expected that every target molecule undergoes either ionization or fragmentation; thus, differences in the parent ion yields at saturation must be due to fragmentation. The precise details of intense-field fragmentation is beyond the scope of this work, though fragmentation due to VUV absorption has been studied elsewhere. ${ }^{36}$ Nevertheless, for the purposes of this work it is useful to identify general trends, such as the relative instability of the singly ionized pyrimidine parent and the relative enhancement of the singly ionized pyridazine parent. Oddly, these two are the most similar in their electronic orbital configuration, both having their antisymmetric $n$ and $\pi$ orbitals as the HOMO and HOMO-1, respectively.

Each of the molecules presented is also found in its dicationic state. While the precise dynamics of the second ionization cannot be inferred through the results of the current experiment, we note that pyrazine has a larger propensity to form a doubly charged ion than the other species. We attribute this to the fact that pyrazine's symmetry $\left(D_{2 h}\right)$ differentiates it from the rest of the series $\left(\mathrm{C}_{2 \mathrm{v}}\right)$ and gives it a symmetric $\left(\mathrm{A}_{\mathrm{g}}\right) \mathrm{HOMO},{ }^{37,38}$ though why this leads to a more stable dication needs further investigation. The doubly-charged parent ions of each species have approximately the same appearance intensity $\left(\approx 6-8 \times 10^{13} \mathrm{~W} / \mathrm{cm}^{2}\right)$, but the yield for pyrazine rises more prominently than the rest. In fact, the pyrazine dication yield rises with a log-log slope of six, although due to the more complicated dynamics of multiple ionizations, this is not likely indicative of a six-photon absorption.

We also observe that the pyridine dication yield is suppressed in comparison to the rest of the series, even saturating between 3-4 $\times 10^{14} \mathrm{~W} / \mathrm{cm}^{2}$. This suggests an inherent instability in the multiply-charged parent ion of pyridine in comparison to the other species; this is further upheld by the appearance of broader features in the mass spectra suggestive of long-lived metastable states which fragment during their flight to the detector. ${ }^{39}$ The details of this process are not 
presented here as they are the subject of further work and beyond the primary scope of the current paper. Pyridine shows a large (multiple orders of magnitude) enhancement in the yield of such broad features in the mass spectra, which adds credence to the sort of instability seen in the dication.

\section{CONCLUSIONS}

While the $\pi$ system inherited from benzene is only mildly perturbed, and thus capable of playing a strong role in the intense-field dynamics of the mono- and di-substituted azabenzenes, the presence of lone pair states allows for much richer dynamics. In particular, the number of possible transitions in competition is increased compared to benzene and, in some cases, it is these transitions that dominate the intensefield ionization dynamics.

In particular, it seems that the introduction of the $n$ state in pyridine does little to perturb the dynamics compared to benzene other than to destabilize the dication. It is only in the diazines, where multiple lone pairs interact with each other, that the dynamics becomes significantly perturbed. In pyrazine, where the symmetric superposition of lone pair orbitals becomes the HOMO, the perturbation affects the system differently. This sets pyrazine apart from the other species in terms of different ionization dynamics and increased yield of the dicationic state.

Field-induced molecular alignment studies ${ }^{40}$ often operate under similar laser conditions as the present work, although typically the laser intensity is kept slightly below the ionization threshold. In light of the present work, molecules with similar nitrogen heterocyclic systems may be poor choices for alignment studies; the increased number of transitions resulting from the lone pairs may lead to considerable excitation even below the ionization threshold, meaning that the aligned molecules may be in a variety of excited states when measured. Selection of molecular targets in such studies should be made carefully in light of the present work.

\section{EXPERIMENTAL}

We used 800-nm ( $\hbar \omega=1.55 \mathrm{eV}), 50$-fs pulses produced by a Ti:sapphire laser system based on chirped-pulse amplification. These pulses were focused into a target vacuum chamber at stagnation pressures of $\sim 1 \times 10^{-7} \mathrm{mbar}$, with this pressure chosen to maximize the count rate while avoiding distortions in the data. The resulting Gaussian laser focus, as measured with beam imaging software, was found to have a waist of $35 \mu \mathrm{m}$ in FWHM in intensity. To minimize intensity gradients and chromatic focusing aberrations we collected ions not from the focal waist, but from a volume $3 \mathrm{~mm}$ away on the defocusing side. Intensities were calibrated by comparing saturation intensities in the current alignment scheme to that of fluorobenzene, which had previously ${ }^{21}$ been calibrated to the well-documented saturation intensity of atomic xenon. Rotating a half-wave plate in the laser's compressor allowed adjustable attenuation of the beam ranging from $\sim 8 \times 10^{12} \mathrm{~W} / \mathrm{cm}^{2}$ to $\sim 5 \times 10^{14} \mathrm{~W} / \mathrm{cm}^{2}$. This range of intensities was adequate for identifying the relevant processes in all molecules considered.
Ions were detected with a double microchannel plate, the signal of which was fed into a counting card controlled by a PC. The spectrometer was tuned to map the spatial ion densities created by the laser pulses in the focus onto the TOF spectrum; by setting a region of interest in the TOF spectrum we then count ions created in a micrometer-sized, limited detection volume only. Inside this detection volume the intensity is nearly constant. Ion counts from outside the detection volume are simply discarded. Further details of the three-dimensional spatial ion resolution can be found in Ref. 23. For the present measurements we adjusted the dimensions of the detection volume to $500 \mu \mathrm{m}$ (along the laser propagation direction) $\times 15 \mu \mathrm{m} \times 15 \mu \mathrm{m}$ (along the two transverse directions). We estimate that the local intensity variations inside the detection volume are less than $14 \%$; thus, the intensity is uniform up to $1 / 18$ th of a decade. This estimate includes possible vibrations and laser pointing variations of up to $\pm 4 \mu \mathrm{m}$ standard deviation. The intrinsic fluctuations in the laser pulse energy were less than $8 \%$. All target chemicals were obtained from Aldrich, with purity 99\% or better and used as purchased, with the exception of pyridazine, which was $98 \%$. The pyridazine sample was dried over $\mathrm{KOH}$ and distilled under reduced pressure for purification; ion yield measurements were taken both before and after purification with no discernable differences.

\section{ACKNOWLEDGMENTS}

This material is based upon work supported by the National Science Foundation (NSF) under Grant No. PHY0855675. The authors gratefully acknowledge the Max Planck Institute of Quantum Optics in Germany, in particular Dr. Hartmut Schröder, for generously lending us the reflectron used in this work. The authors wish to thank Dr. Steven DiMagno for purifying the pyridazine sample.

\footnotetext{
${ }^{1}$ J. McMurry, Organic Chemistry (Brooks/Cole, Belmont, MA, 2008).

${ }^{2}$ A. Ishii and Y. Katsumata, in Drugs and Poisons in Humans, edited by O. Suzuki and K. Watanabi (Springer, New York, 2005), Vol. 2, p. 591.

${ }^{3} \mathrm{~T}$. Eicher and S. Hauptmann, The Chemistry of Heterocycles (Wiley-VCH, Weinheim, 2003).

${ }^{4}$ I. Walker, M. Palmer, and A. Hopkirk, Chem. Phys. 141, 365 (1990).

${ }^{5}$ M. Palmer and I. Walker, Chem. Phys. 157, 187 (1991).

${ }^{6}$ M. Palmer, I. Walker, M. Guest, and A. Hopkirk, Chem. Phys. 147, 19 (1990).

${ }^{7}$ I. Walker and M. Palmer, Chem. Phys. 153, 169 (1991).

${ }^{8}$ M. Riese, Z. Altug, and J. Grotemeyer, Phys. Chem. Chem. Phys. 8, 4441 (2006).

${ }^{9}$ K. Choi, D. Alm, J. Lee, and S. Kim, J. Phys. Chem. A 110, 2634 (2006).

${ }^{10}$ S. Sato, K. Omiya, and K. Kimura, J. Electron Spectrosc. Relat. Phenom. 97, 121 (1998)

${ }^{11}$ M. de Groot and W. J. Buma, J. Chem. Phys. 127, 104301 (2007).

${ }^{12}$ M. Stener, P. Decleva, D. M. P. Holland, and D. A. Shaw, J. Phys. B 44, 075203 (2011).

${ }^{13}$ K. Innes, I. Ross, and W. Moomaw, J. Mol. Spectrosc. 132, 492 (1988).

${ }^{14}$ P. Corkum, Phys. Rev. Lett. 71, 1994 (1993).

${ }^{15}$ A. Becker and F. H. M. Faisal, J. Phys. B 38, R1 (2005).

${ }^{16}$ I. V. Hertel and W. Radloff, Rep. Prog. Phys. 69, 1897 (2006).

${ }^{17}$ Z. Chang, Fundamentals of Attosecond Optics (CRC, Boca Raton, 2011).

${ }^{18}$ W. Boutu, S. Haessler, H. Merdji, P. Breger, G. Waters, M. Stankiewicz, L. J. Frasinski, R. Taieb, J. Caillat, A. Maquet, P. Monchicourt, B. Carre, and P. Salieres, Nat. Phys. 4, 545 (2008).

${ }^{19}$ H. Rabitz, Science 314, 264 (2006).

${ }^{20}$ Methods are beyond reach (see http://www.physorg.com/news194276894. html). Though impressive, this research required immense and costly
} 
resources on a record supercomputer, with software developed and specifically optimized for its architecture. No resources exist to simulate the intense-field photodynamics of larger molecules.

${ }^{21}$ T. D. Scarborough, J. Strohaber, D. B. Foote, C. J. McAcy, and C. J. G. J. Uiterwaal, Phys. Chem. Chem. Phys. 13, 13783 (2011).

${ }^{22}$ D. Cardoza, B. J. Pearson, and T. Weinacht, J. Chem. Phys. 126, 084308 (2007).

${ }^{23}$ J. Strohaber and C. J. G. J. Uiterwaal, Phys. Rev. Lett. 100, 023002 (2008).

${ }^{24}$ M. Walker, P. Hansch, and L. Van Woerkom, Phys. Rev. A 57, R701 (1998).

${ }^{25}$ T. Goodworth, W. Bryan, I. Williams, and W. Newell, J. Phys. B 38, 3083 (2005).

${ }^{26}$ L. Robson, K. Ledingham, P. Mckenna, T. Mccanny, S. Shimizu, J. Yang, C. Wahlstrom, R. Lopez-Martens, K. Varju, P. Johnsson, and J. Mauritsson, J. Am. Soc. Mass Spectrom. 16, 82 (2005).

${ }^{27}$ J. Strohaber, A. A. Kolomenskii, and H. A. Schuessler, Phys. Rev. A 82, 013403 (2010).

${ }^{28}$ F. H. M. Faisal, in Lectures on Ultrafast Intense Laser Science, edited by K. Yamanouchi (Springer, New York, 2010), Vol. 1, p. 1.

${ }^{29}$ L. Keldysh, Sov. Phys. JETP 20, 1307 (1965).

${ }^{30}$ The ionization suppression at low intensity is repeatable through several datasets subjected to a wide array of experimental parameters, yet remains unique to pyridine, though it must be emphasized that the lowest intensities have the largest relative uncertainties in both intensity calibration and counting statistics. If one were to assume the suppression to be physical, one could imagine that the dominant excitation transition is slightly off resonance until the rising edge of the laser pulse is strong enough to reinforce the resonance through a dynamical Stark shift, known as a dynamic resonance. Though our data alone cannot support this, it could serve as a plausible hypothesis.

${ }^{31}$ C. Hannay, D. Duflot, J. Flament and M. Hubin-Franskin, J. Chem. Phys. 110, 5600 (1999).

${ }^{32}$ NIST WebBook (http://webbook.nist.gov/chemistry) lists both vertical and adiabatic ionization energies for each of the azabenzenes; the difference in these values is never greater than $\approx 0.6 \mathrm{eV}$.

${ }^{33}$ N. V. Bordyug and V. P. Krainov, Laser Phys. Lett. 4, 418 (2007).

${ }^{34}$ A. Bolovinos, P. Tsekeris, J. Philis, E. Pantos, and G. Andritsopoulos, J. Mol. Spec. 103, 240 (1984).

${ }^{35}$ R. W. Boyd, Nonlinear Optics, 2nd ed. (Elsevier, San Diego, 2003), Sec. 12.5 .

${ }^{36}$ G. Vall-llosera, M. Coreno, P. Erman, M. A. Huels, K. Jakubowska, A. Kivimäki, E. Rachlew, and M. Stankiewicz, Int. J. Mass Spectrom. 275, 55 (2008).

${ }^{37}$ F. A. Cotton, Chemical Applications of Group Theory (Wiley, New York, 1963).

${ }^{38}$ D. C. Harris and M. D. Bertolucci, Symmetry and Spectroscopy (Dover, New York, 1978).

${ }^{39}$ U. Boesl, H. Neusser, R. Weinkauf, and E. Schlag, J. Phys. Chem. 86, 4857 (1982).

${ }^{40}$ H. Stapelfeldt and T. Seideman, Rev. Mod. Phys. 75, 543 (2003). 